\title{
CUL4A promotes proliferation and metastasis of colorectal cancer cells by regulating H3K4 trimethylation in epithelial-mesenchymal transition
}

\author{
This article was published in the following Dove Press journal: \\ OncoTargets and Therapy \\ 9 February 2017 \\ Number of times this article has been viewed
}

\begin{abstract}
Xuemei Sui, ${ }^{1, *}$ Hong Zhou, ${ }^{2, *}$ Lei Zhu, ${ }^{3}$ Deqiang Wang, ${ }^{4}$ Sumei Fan, ${ }^{5}$ Wei Zhao ${ }^{6}$

'Clinical Laboratory, The First Affiliated Huai'an Hospital of Nanjing Medical University, ${ }^{2}$ Huai'an No 4 People's Hospital, Huai'an, ${ }^{3}$ Department of Digestive System, Jiangsu Province Hospital of TCM, Nanjing, ${ }^{4}$ Cancer Therapy Center, Affiliated Hospital of Jiangsu University, Zhenjiang, ${ }^{5}$ Geriatric Department, The Affiliated Huai'an Hospital of Xuzhou Medical University, Huai'an, ${ }^{6}$ Clinical Laboratory, Obstetrics and Gynecology Hospital Affiliated to Nanjing Medical University, Nanjing, Jiangsu, People's Republic of China

*These authors contributed equally to this work
\end{abstract}

Correspondence: Sumei Fan Geriatric Department, The Affiliated Huai'an Hospital of Xuzhou Medical University, 62 South Huaihai Road, Qingpu, Huai'an, Jiangsu 22300I, People's Republic of China

Tel +86 I39 I5 II 88II

Email fansmxzmu@163.com

Wei Zhao

Clinical laboratory, Obstetrics and Gynecology Hospital Affiliated to Nanjing Medical University, 123 Tianfeixiang, Mochou Road, Qinhuai, Nanjing, Jiangsu 210004 , People's Republic of China Tel +86 I36 0l 468652

Email weizhao_smart00।@I26.com

\begin{abstract}
Increasing evidence suggests that CUL4A, a ubiquitin ligase, is involved in the promotion of cancer malignancy and correlated with worse clinical prognosis in several kinds of human cancers. Although its effect and mechanism on the progression of colorectal cancer (CRC) remain unknown. Our clinical data show that CUL4A protein is overexpressed, positively associated with lymph nodes status, differentiation degree, tumor size, and poor prognosis in $80 \mathrm{CRC}$ patients. CUL4A overexpression promotes cell proliferation and colony formation of CRC cells. Knockdown of CUL4A inhibits cell proliferation and migration. CUL4A can significantly promote the in vitro migration of CRC cells via induction of the epithelial-mesenchymal transition process. And the modulation of CUL4A expression altered the level of $\mathrm{H} 3 \mathrm{~K} 4$ trimethylation at the E-cadherin, N-cadherin, and vimentin gene promoters, which in turn transcriptionally regulated their expression. Moreover, knockdown of CUL4A also decreased the tumor volume and tumor weight in vivo. Together, our results reveal that CUL4A plays as an oncogene in CRC and may become a potential therapeutic target in the treatment of colorectal cancer.
\end{abstract}

Keywords: colorectal cancer, CUL4A, EMT, migration, H3K4 trimethylation

\section{Introduction}

In 2013, colorectal cancer (CRC) was the third most common cancer and the fourth leading cause of death worldwide. ${ }^{1}$ An increasing number of oncogenes have been reported to be responsible for the development of CRC, such as S100P, K-Ras, BRAF, visfatin, and pinin. ${ }^{2-6}$ Primary CRC derives from the epithelial cells of the gastrointestinal tract. ${ }^{7}$ During epithelial-mesenchymal transition (EMT) progression, cancer cells are thought to acquire a phenotype of the mesenchymal cell, which allows them to lose their junctions, invade surrounding tissues, and migrate to distant organs. ${ }^{7}$ EMT is initiated by a set of transcription factors, such as Snail, Twist1, and ZEB, which execute EMT by repressing epithelial genes and activating mesenchymal genes. ${ }^{8}$ $\mathrm{H} 3 \mathrm{~K} 4$ trimethylation $(\mathrm{H} 3 \mathrm{~K} 4 \mathrm{me} 3)$ is a prevalent mark that is exclusively associated with actively transcribed genes, whereas trimethylation of H3K27 (H3K27me3) is associated with gene repression. ${ }^{9}$

CUL4A is a member of the evolutionally conserved cullin family, which is evolutionally conserved and includes seven-related cullins (Cul1, Cul2, Cul3, Cul4A, Cul4B, Cul5, and Cul7). ${ }^{10}$ CUL4A constitutes the ubiquitin ligase E3 complex, and plays crucial 
roles in DNA replication, cell cycle regulation, and genomic instability. ${ }^{11-15}$ Previous studies have shown that CUL4A has been reported to have an essential role in the ubiquitination of several well-defined tumor suppressor genes, for instance p21, p27, p53, and DDB2, which provides evidence that CUL4A might be a potential oncogene. ${ }^{15-18}$ CUL4A overexpression has been discovered in several kinds of human cancers, including breast cancer, prostate cancer, non-small cell lung cancer (NSCLC), and malignant pleural mesothelioma (MPM). ${ }^{19-23}$

In this study, we provide evidence that CUL4A was overexpressed in primary CRC and cell line HCT-116 and predicted poor overall patient survival. Moreover, CUL4A promoted cell proliferation, colony formation, and migration of HCT-116. We also found that CUL4A promoted CRC development through EMT pathway and CUL4A triggered the EMT process via regulation of $\mathrm{H} 3 \mathrm{~K} 4 \mathrm{me} 3$. Finally, knockdown of CUL4A inhibited tumor size and weight in vivo. In conclusion, these data suggest that CUL4A is a potential novel target for CRC diagnosis and therapy.

\section{Materials and methods}

\section{Patients and specimens}

Primary CRC tissues and their corresponding adjacent normal tissues were obtained from the CRC patients treated in the Affiliated Huai'an Hospital of Xuzhou Medical University. The study was approved by the Medical Ethics Committee of the Affiliated Huai'an Hospital of Xuzhou

Table I Association between CUL4A expression and clinicopathologic features of colorectal cancer patients

\begin{tabular}{|c|c|c|c|c|}
\hline \multirow[t]{2}{*}{ Variables } & \multirow[t]{2}{*}{ Patients (n) } & \multicolumn{3}{|c|}{ CUL4A expression } \\
\hline & & $\begin{array}{l}\text { Student's } \\
\text { t-test }\end{array}$ & $x^{2}$ & $P$-value \\
\hline Gender & & & 0.235 & 0.628 \\
\hline Male & 44 & 35 & & \\
\hline Female & 36 & 27 & & \\
\hline Age (years) & & & 0.346 & 0.556 \\
\hline$\leq 50$ & 29 & 21 & & \\
\hline$>50$ & 51 & 35 & & \\
\hline Tumor size (cm) & & & 11.255 & $0.00 \mathrm{I}^{\text {*** }}$ \\
\hline$\leq 2$ & 27 & 15 & & \\
\hline$>2$ & 53 & 47 & & \\
\hline Differentiation degree & & & 4.891 & $0.049 *$ \\
\hline High differentiation & 32 & 20 & & \\
\hline Low/medium differentiation & 48 & 42 & & \\
\hline Lymph nodes status & & & 6.882 & 0.009 ** \\
\hline Negative & 34 & 18 & & \\
\hline Positive & 46 & 44 & & \\
\hline TNM grade & & & 0.478 & 0.489 \\
\hline$|-| \mid$ & 30 & 22 & & \\
\hline III-IV & 50 & 40 & & \\
\hline
\end{tabular}

Note: $* P \leq 0.05 ; * * P \leq 0.01 ; * * * P \leq 0.00$ I.

Abbreviation: TNM, tumor node metastasis.
Medical University and written informed consent was obtained from all the patients involved in this study. Detailed clinical histopathologic factors were presented in Table 1. For the measurement of prognosis, we analyzed the clinical data overall survival (OS), defined as the time from surgery to death. All recruited patients had been followed-up periodically until the patients death.

\section{Antibodies and Western blot}

$\mathrm{H} 3 \mathrm{~K} 4 \mathrm{me} 3$ rabbit mAb, E-cadherin mouse mAb, N-cadherin rabbit $\mathrm{mAb}$, and $\beta$-actin mouse $\mathrm{mAb}$ were purchased from Cell Signaling Technology (Danvers, MA, USA). The cells were lysed in a buffer containing $20 \mathrm{mM}$ Tris- $\mathrm{HCl}(\mathrm{pH} 7.5)$, $150 \mathrm{mM} \mathrm{NaCl}, 5 \mathrm{mM}$ ethylene glycol-bis( $\beta$-aminoethyl ether)- $N, N, N^{\prime}, N^{\prime}$-tetraacetic acid, and $1 \%$ Nonidet P 40 with a mixture of protease inhibitors before Western blot assay.

\section{Immunohistochemistry (IHC) analysis}

The CRC patients specimens were fixed in 4\% paraformaldehyde solution overnight and embedded in paraffin wax the next day. For histologic analysis, sections were cut at a thickness of $4 \mu \mathrm{m}$ and followed by hematoxylin and eosin staining. All sections were processed for IHC using an ABC kit (Vector Laboratories, Burlingam, CA, USA) according to the manufacturer's instruction and observed under a microscope (Olympus Corporation, Tokyo, Japan). Representative photographs were taken.

\section{Cell culture, lentivirus packaging, and infection}

HEK293T and human CRC cell line HCT-116 were purchased from Bank/Stem Cell Bank, Shanghai Institute for Biological Sciences, Chinese Academy of Sciences (Shanghai, People's Republic of China). HEK293T cells were cultured in Dulbecco's Modified Eagle's Medium (DMEM; Thermo Fisher Scientific, Waltham, MA, USA) containing 10\% fetal bovine serum (FBS; Thermo Fisher Scientific). Then HCT-116 cells were cultured in Roswell Park Memorial Institute 1640 (Thermo Fisher Scientific) supplemented with $10 \%$ FBS.

For lentivirus packaging, HEK293T cells $\left(7 \times 10^{6}\right)$ were plated in a $15 \mathrm{~cm}$ dish, incubated for 24 hours (h), and then transfected with $15 \mu \mathrm{g}$ of lentivirus plasmids. After $48 \mathrm{~h}$, the virus containing medium was filtered through a $0.45 \mu \mathrm{m}$ filter (EMD Millipore, Billerica, MA, USA) and collected as the first supernatant. Additional DMEM medium with FBS was added into the dish and the virus-containing medium was filtered and collected as the second supernatant after another $24 \mathrm{~h}$. Both of the first and second supernatant was 
then centrifuged at $20,000 \times g$ for $2 \mathrm{~h}$. The supernatant was abandoned and the precipitate was resuspended in $100 \mu \mathrm{L}$ DMEM without FBS.

For infection, gradient virus-contained DMEM was added with polybrene (Sigma-Aldrich Co., St Louis, MO, USA, $4 \mu \mathrm{g} / \mathrm{mL}$ ), and then the culture dishes were incubated at $37^{\circ} \mathrm{C}$ for $6 \mathrm{~h}$ and replaced by fresh medium. After incubation for $36-48 \mathrm{~h}$, the infected cell populations were confirmed by fluorescence microscope for green fluorescent protein expression to evaluate the virus titer. Target cells were plated in a 6-well plate for infection by appropriate volume of virus-contained DMEM.

\section{RNA isolation and quantitative reverse transcription-polymerase chain reaction (RT-PCR)}

Total RNA was extracted from cells by Trizol reagent (Thermo Fisher Scientific) then reverse transcribed and synthesized to cDNA using avian myeloblastosis virus reverse transcriptase (Takara, Dalian, People's Republic of China) according to these manufacturers' instructions. The quantification of gene transcripts was determined by real-time PCR using SYBR Green Real time PCR Master Mix (Toyobo) and Mx3000P quantitative PCR (qPCR) system (Stratagene, La Jolla, LA, USA). Glyceraldehyde 3-phosphate dehydrogenase (GAPDH) was used as the internal control. The qPCR primers are 5'-CTCCAAGAAGCTGGTCATCA-3' and 5'-GAGCTCCTCGAGGTTGTACC-3' for CUL4A; 5' - TACACTGCCCAGGAGCCAGA-3' and 5'-TGGCACCAGTGTCCGGATTA-3' for E-cadherin; 5'-GACGGTTCGCCATCCAGAC-3' and 5'-TCGATTGGTTTGACCACGG-3' for N-cadherin; 5' - T G A G T A C C G G A G A C A G G T G C A G - 3' and 5'-TAGCAGCTTCAACGGCAAAGTTC-3' for vimentin; 5'-GCACCGTCAAGGCTGAGAAC-3' and 5'-TGGTGAAGACGCCAGTGGA-3' for GAPDH.

\section{Colony formation and wound healing assays}

For colony formation assay, the HCT-116 cells were seeded in to a 6 -well plate (400 cells/well). After 5 days, the colonies were washed with $1 \times$ phosphate-buffered saline (PBS) and stained with crystal violet for $20 \mathrm{~min}$, then imaged and quantified.

For wound-healing assay, HCT- 116 cells $\left(5 \times 10^{5}\right.$ cells/well $)$ were seeded into a 6-well plate. After incubation for $24 \mathrm{~h}$, a wound was created by scratching the confluent monolayer layer with a yellow tip. After washing with $1 \times$ PBS three times, cells were incubated with serum-free medium.
Images were taken immediately and $24 \mathrm{~h}$ later under a microscope (Olympus Corporation).

\section{Chromatin immunoprecipitation (ChIP) and PCR detection}

ChIPs were performed using the ChIP Assay Kit (Upstate Biotechnology, Lake Placid, NY, USA) and the H3K4me3 antibody (9751) from Cell Signaling Technology. Primers used for PCR detection are listed as follows: 5'-CCCGCCCCAGCTGTGTCATTTT-3' and 5'-AATGGTGCCCATCCACGTGG-3' for E-cadherin $(-80$ to +88$)$; 5'-CCAAAGTGCTGGTATTCCGCTGTA AG-3' and 5'-GTGTGCTCCCAGAGTCGGGTTTGC-3' for $\mathrm{N}$-cadherin $(-5,112$ to $-4,961), 5^{\prime}$-GGTGTGGTT TCATGGGGGGAGG-3' and 5'-CCCTAAGTTTTTAATA ACTCGCTAAAG-3' for vimentin $(-116$ to +91$)$.

\section{Nude mice xenograft model}

The animal study was performed in strict accordance with the National Institutes of Health, Guide for the Care and Use of Laboratory Animals. All the animal experiments were approved by the institutional review board of The Affiliated Huai'an Hospital of Xuzhou Medical University. The nude mice (4 weeks old, male) were randomly divided into two groups (n=6/group). Two HCT-116 cell lines were established, including CUL4A knockdown cell line and its control cell line. The cells in the logarithmic phase of growth were trypsinized and rinsed with $1 \times$ PBS three times. Each nude mouse was injected with HCT-116 cells $\left(5 \times 10^{6}\right.$ cells $)$ in 100 $\mu \mathrm{L}$ of $1 \times$ PBS in the upper right shoulder subcutaneously. Tumor sizes were measured by measuring two perpendicular diameters with digital calibers every 5 days, and tumor volume was calculated using the equation: $\left(\right.$ Length $\left.\times \mathrm{Width}^{2}\right) / 2$.

\section{Statistical analysis}

All data were expressed as the mean \pm standard deviation from three independent experiments. Student's $t$-test was used to evaluate the data. $\chi^{2}$ test was used to analyze the differences between groups of CUL4A expression in CRC tissues. The log-rank test was used to explore the association between CUL4A expression and the OS of CRC patients. SPSS version 19.0 was adopted for clinical data analysis. Values were considered to be statistically significant at $P<0.05$ and $P<0.01$.

\section{Results CUL4A is upregulated in primary CRC tissues}

To explore the potential impact of CUL4A in CRC, we examined CUL4A expression in $80 \mathrm{CRC}$ tissues and 
A

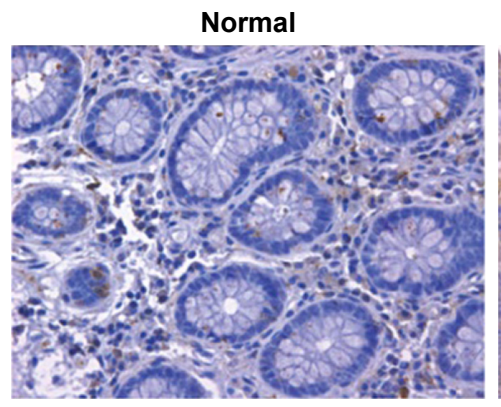

B

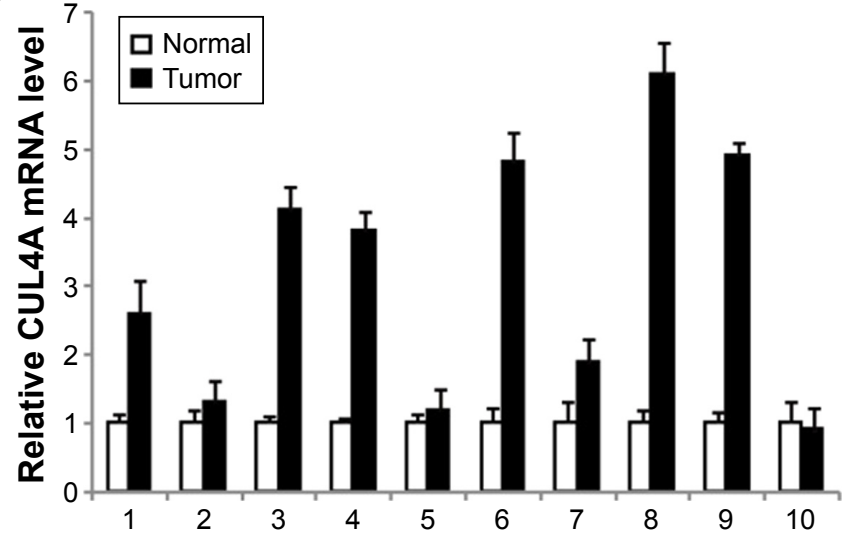

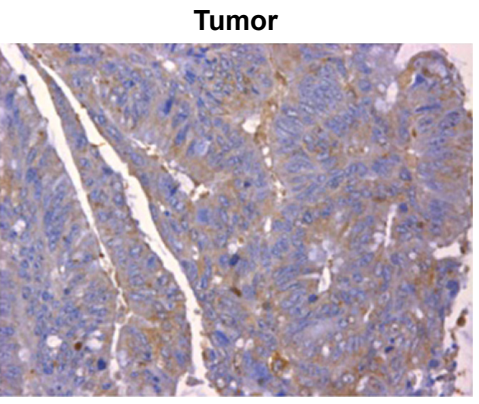

C

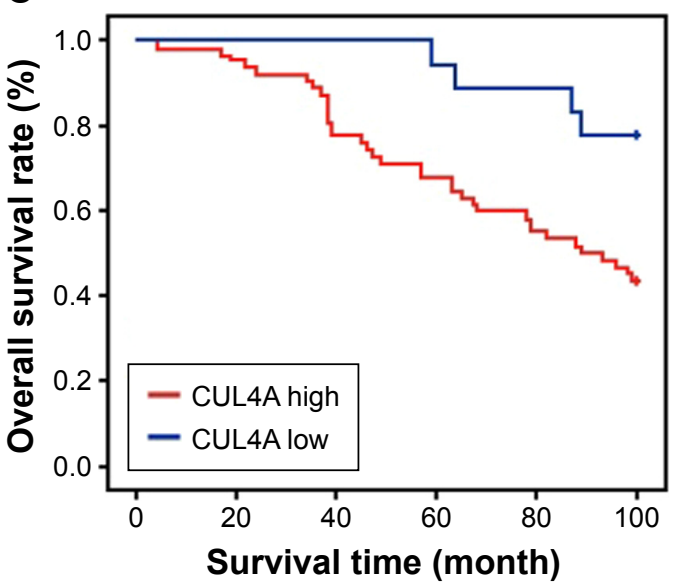

Figure I CUL4A is overexpressed and associated with prognosis in colorectal cancer.

Notes: (A) The expression of CUL4A was detected in colorectal normal tissue and cancer tissue by immunohistochemistry, and representative samples are shown at $400 \times$ magnification. (B) Comparison of mRNA expression levels of CUL4A in I0 pairs of tumor and adjacent normal samples with qRT-PCR. (C) The overall survival rate of the 80 colorectal cancer patients were compared in the CUL4A low and CUL4A high groups. Statistical significance was determined using the log-rank test. Abbreviation: qRT-PCR, quantitative reverse transcription-polymerase chain reaction.

30 normal tissues by IHC. CUL4A expression was significantly increased in CRC tissues compared with normal tissues (Figure 1A). Next, we investigated the mRNA level of CUL4A in primary CRC by quantitative RT-PCR (qRTPCR). The mRNA level of CUL4A was determined in tumors and their corresponding paracancerous histologic normal tissue from 10 clinically and pathologically annotated cases of CRC. The results demonstrated that CUL4A was robustly increased in 70\% (7 of 10) of CRC (Figure 1B).

Next, Kaplan-Meier survival analysis was conducted to investigate the correlation between CUL4A expression and $\mathrm{CRC}$ patient prognosis. The patients were divided into two groups with high expression or low expression level of CUL4A. The Kaplan-Meier survival test revealed that CRC patients with high CUL4A expression had shorter OS than those patients with low CUL4A expression $(P=0.001$; Figure 1C). The expression level of CUL4A was correlated significantly with the OS of CRC patients.

With clinicopathologic correlation analysis, we found that CUL4A protein level was positively correlated with lymph nodes status, differentiation degree, tumor size band poor prognosis in $80 \mathrm{CRC}$ patients (Table 1).

\section{CUL4A promotes cell proliferation and colony formation}

To detect the role of CUL4A in CRC, we created a CUL4A gain of function in CRC cells. Overexpression of CUL4A in HCT-116 cell was performed by a lentivirus-based method. The mRNA level of CUL4A in these cells was determined by qRT-PCR (Figure 2A). The cell proliferation rate of these cells was investigated by 3-(4,5-dimethylthiazol-2-yl)-2, 5-diphenyltetrazolium bromide (MTT) assay, and CUL4A overexpression significantly increased the cell growth rate (Figure 2C). The knockdown of CUL4A was performed by a lentivirus-based method. A control short hairpin RNA (shRNA) or CUL4A-specific shRNA was introduced into HCT-116 cells. As confirmed by qRT-PCR, the mRNA level of CUL4A was successfully reduced by a CUL4A-specific shRNA (Figure 2B). Knockdown of CUL4A decreased cell proliferation rate of HCT-116 cells as shown by MTT assay (Figure 2D).

Next, colony formation was used to investigate the potential of tumorigenesis of HCT-116 cells. We found that CUL4A overexpression significantly promoted colony formation in these cells (Figure 2E), whereas the capacity of colony formation was reduced by CUL4A knockdown (Figure 2F). 
A

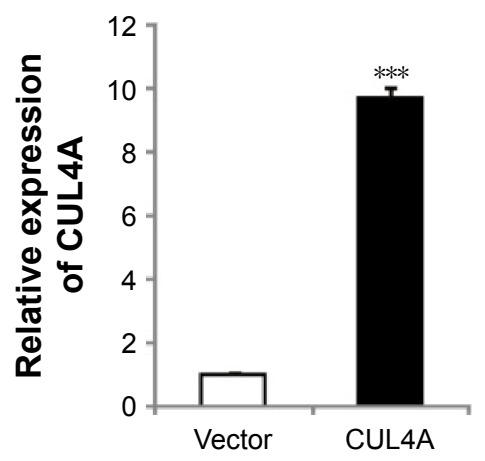

C

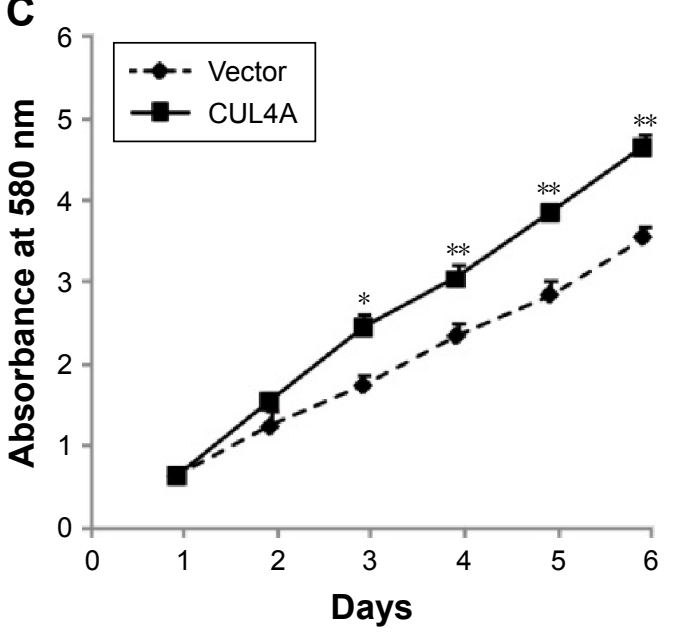

$\mathbf{E}$
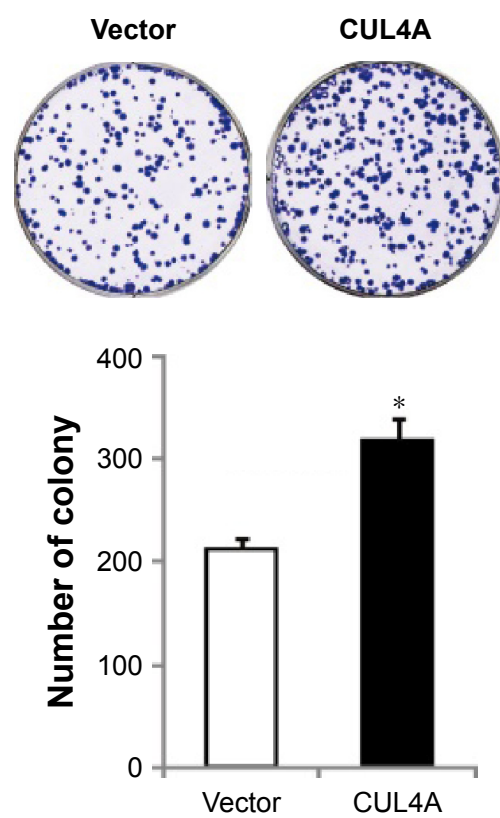

B

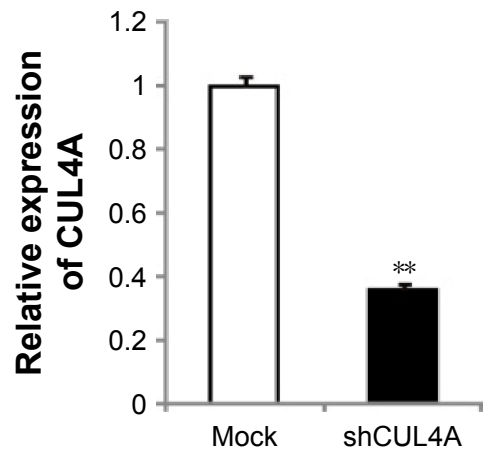

D

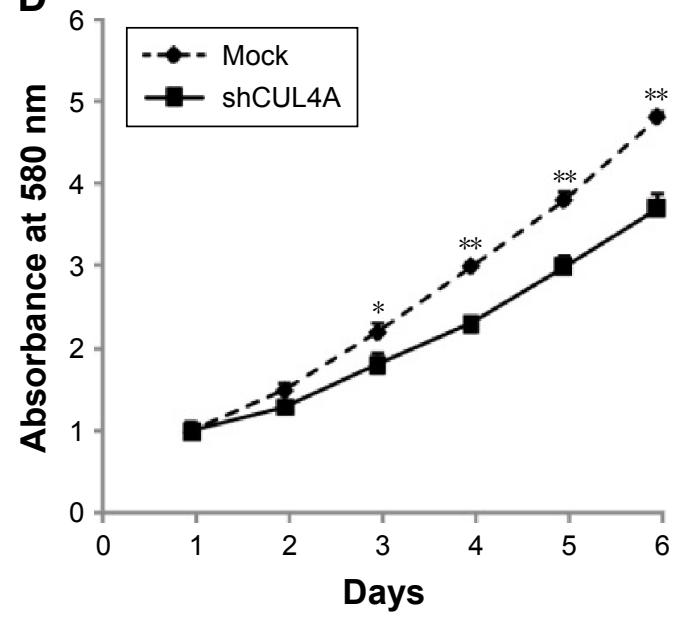

$\mathbf{F}$
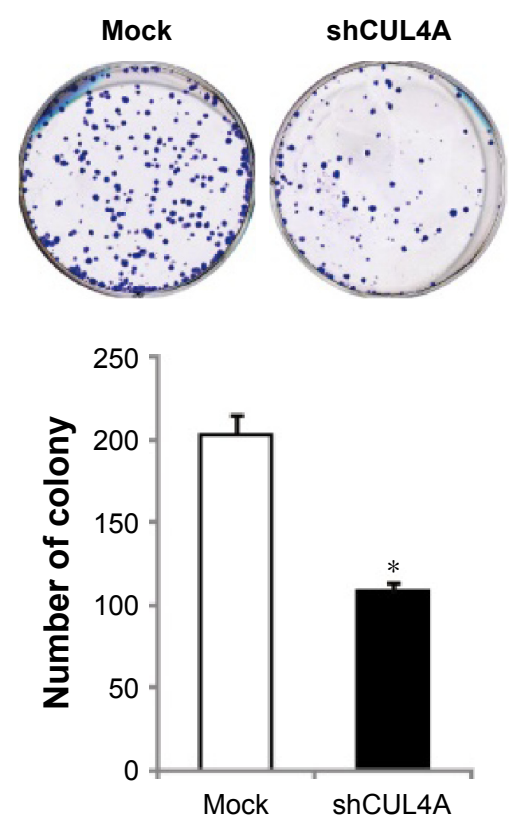

Figure 2 CUL4A promotes colorectal cancer cell line HCT-1 16 growth and migration.

Notes: (A) The mRNA level of CUL4A in HCT-116 cells infected with lentivirus containing vector or CUL4A was detected by qRT-PCR. (B) The mRNA level of CUL4A in HCT-1 16 cells infected with lentivirus containing mock or shCUL4A was detected by QRT-PCR. (C) Cell proliferation rate of vector or CUL4A cells were determined by MTT assay at the indicated time point. (D) Cell proliferation rate of mock or shCUL4A cells were determined by MTT assay at the indicated time point. The statistical results are shown in the lower panel. Effect of CUL4A overexpression $(\mathbf{E})$ and knockdown $(\mathbf{F})$ on colony formation were measured in HCT-I 16 cells. The cells were seeded into 6-well plates and cultured for 5 days, followed by crystal violet staining. The colony counts were shown below the graph. All the data are shown as mean \pm standard deviation and $* P \leq 0.05, * * P \leq 0.01, * * * P \leq 0.001$.

Abbreviations: MTT, 3-(4,5-dimethylthiazol-2-yl)-2,5-diphenyltetrazolium bromide; qRT-PCR, quantitative reverse transcription-polymerase chain reaction. 


\section{CUL4A promotes migration of CRC cell line}

Clinical data revealed that CUL4A is positively correlated with lymph node metastasis of CRC patients, we then investigated the effects of CUL4A on the in vitro motility of CRC cells. Overexpression of CUL4A significantly promoted wound closure of HCT-116 cells (Figure 3A). Whereas silencing of CUL4A by its specific shRNA inhibited the wound closure as compared to the control group (Figure 3B). Therefore, these results indicate that in addition to the regulatory impact on cell proliferation, CUL4A has an effect on the migration of CRC cells.

\section{CUL4A regulates EMT of CRC cells}

To further understand the mechanisms by which CUL4A engaged in CRC development and progression, Western blot was conducted to analyze CUL4A overexpression, knockdown, and vector and mock control cells in HCT-116. Overexpression of CUL4A obviously downregulated the expression of epithelial marker E-cadherin, whereas increased the expression of mesenchymal markers N-cadherin and Vimentin. Repression of CUL4A caused the shift in expression of mesenchymal markers Vimentin, N-cadherin to epithelial marker E-cadherin compared with the control cells (Figure 4A). Similarly, results of mRNA expression profiles of EMT markers in HCT-116 cells also confirmed that overexpression of CUL4A triggered EMT. Furthermore, the silencing of CUL4A by shRNA exhibited the opposite effect (Figure 4B). Collectively, these observations showed a critical role of CUL4A in the EMT and metastatic phenotypes of CRC cells.

\section{CUL4A triggers the EMT via regulation of $\mathrm{H} 3 \mathrm{~K} 4 \mathrm{me} 3$}

We then detected how CUL4A regulates EMT at the transcriptional level. Cullin-RING E3 ubiquitin ligase are frequently involved in histone modification. ${ }^{19,24,25}$ To explore whether CUL4A regulates specific histone methylation in CRC cells, H3K4me3, and H3K27me3 were measured after overexpression of CUL4A. For the reason that $\mathrm{H} 3 \mathrm{~K} 4 \mathrm{me} 3$ is associated with transcriptional activation, we tested whether CUL4A overexpression was associated with the $\mathrm{H} 3 \mathrm{~K} 4 \mathrm{me} 3$ modification at the promoters of E-cadherin, N-cadherin, and vimentin in CRC cells. Quantitative ChIP (qChIP) assay was performed in HCT116 cells. We found that CUL4A expression was associated with decreased $\mathrm{H} 3 \mathrm{~K} 4 \mathrm{me} 3$ levels at region -80 to +88 bp of the E-cadherin promoter. In contrast, the $\mathrm{H} 3 \mathrm{~K} 4 \mathrm{me} 3$ levels of $-5,112$ to $-4,961 \mathrm{bp}$ of the N-cadherin promoter and -116 to +91 bp of the vimentin promoter were increased in HCT-116 cells (Figure 4C). Therefore, these data suggest
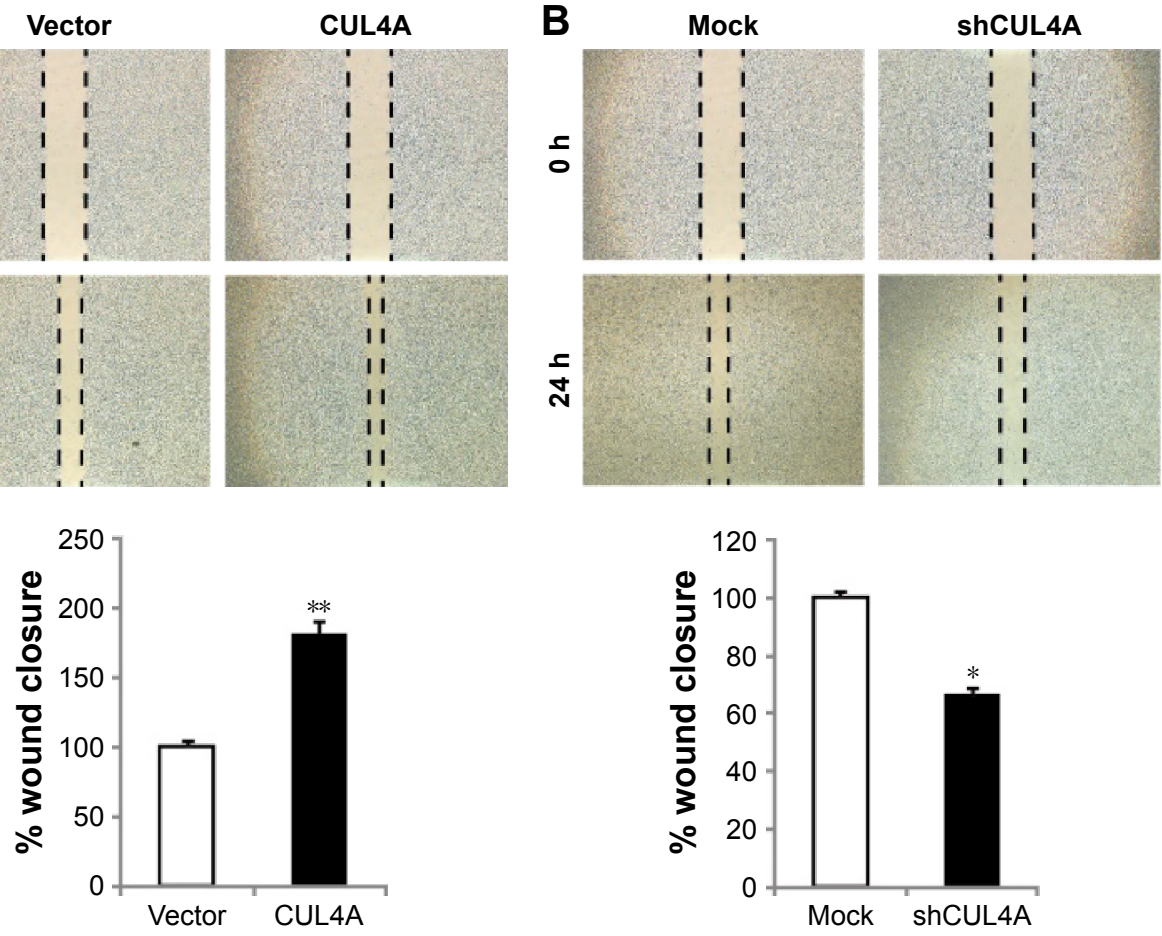

Figure 3 CUL4A promotes migration of colorectal cancer cell line HCT-I 16.

Notes: (A and B) CUL4A overexpressing and knockdown HCT-II 6 cells or control vector cells were subjected to wound healing assay. The statistical results are shown in the lower panel. Data are shown as mean $\pm S D$. $* P \leq 0.05$, **P $\leq 0.01$.

Abbreviations: SD, standard deviation; h, hours. 
A

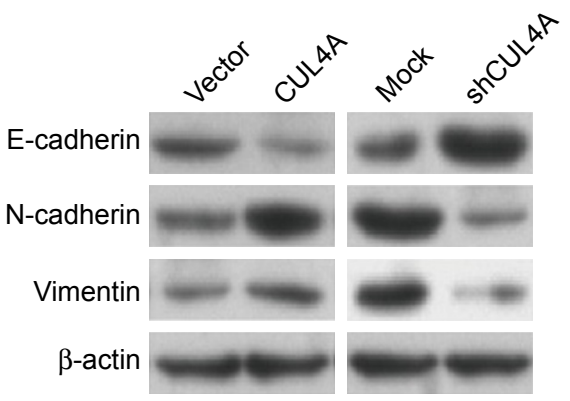

B
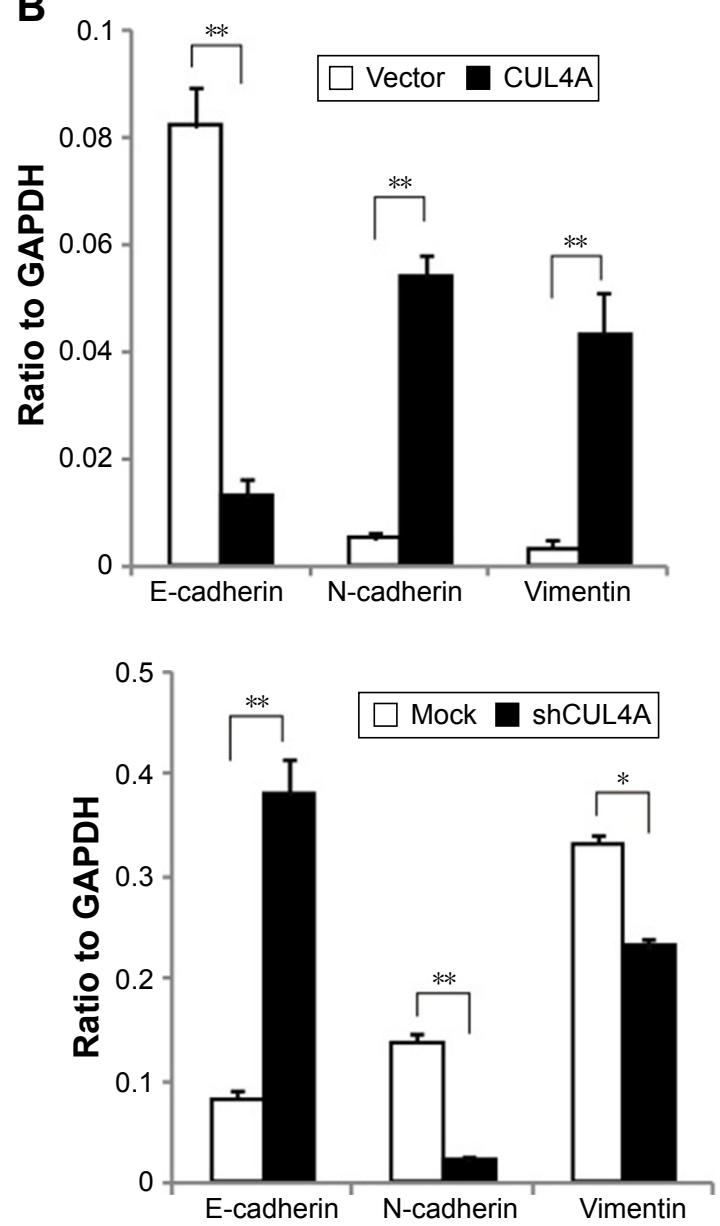

C

E-cadherin promoter
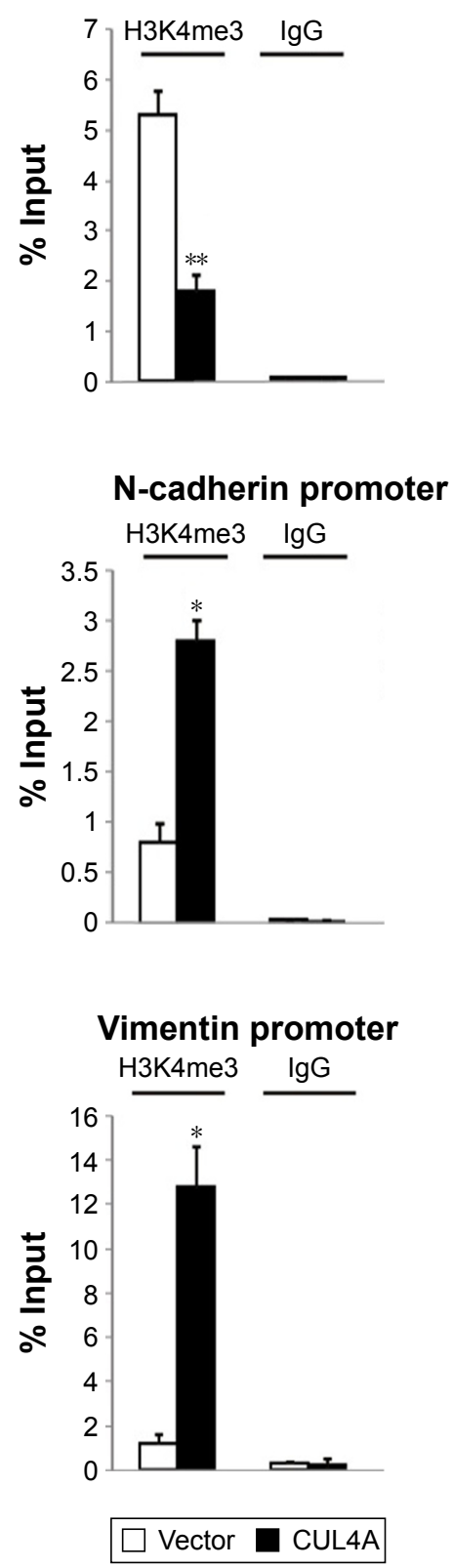

Figure 4 CUL4A promotes epithelial-mesenchymal transition by changing the H3K4me3 level in promoter regions of E-cadherin, $\mathrm{N}$-cadherin and vimentin. Notes: (A and B) Expression of epithelial and mesenchymal markers was analyzed by Western blotting and qRT-PCR. (C) Quantitative chromatin immunoprecipitation was performed to assess H3K4me3 occupancy in HCT-II6 CUL4A overexpression cells. IgG was used as the negative control. "\% input" indicates the ratio of DNA fragment of each promoter region bound by $\mathrm{H} 3 \mathrm{~K} 4 \mathrm{me} 3$ to the total amount of input DNA fragment without $\mathrm{H} 3 \mathrm{~K} 4 \mathrm{me} 3$ antibody pull-down. All the data are shown as mean \pm standard deviation and $* P \leq 0.05, * * P \leq 0.01$.

Abbreviations: GAPDH, glyceraldehyde 3-phosphate dehydrogenase; IgG, immunoglobulin G; H3K4me3, H3K4 trimethylation; PCR, polymerase chain reaction; qRT-PCR, quantitative reverse transcription-polymerase chain reaction.

that CUL4A induces transcriptional activation of $\mathrm{N}$-cadherin and vimentin through regulating $\mathrm{H} 3 \mathrm{~K} 4 \mathrm{me} 3$ and enriching $\mathrm{H} 3 \mathrm{~K} 4 \mathrm{me} 3$ to their promoters.

\section{Knockdown of CUL4A inhibits CRC cell growth in vivo}

To further explore the influence of CUL4A knockdown on CRC cell growth, we investigated the effects of CUL4A on the xenograft model. As evaluated by tumor volumes, knockdown of CUL4A substantially decreased HCT-116 cells growth (Figure 5A). In addition, CUL4A knockdown led to a significant decrease in both tumor size and weight compared with the control cells (Figure 5B and C). Then, we did qRT-PCR to confirm CUL4A expression was knocked down by shCUL4A in these xenograft tumors. The results indicated that CUL4A protein was significantly reduced by 
A

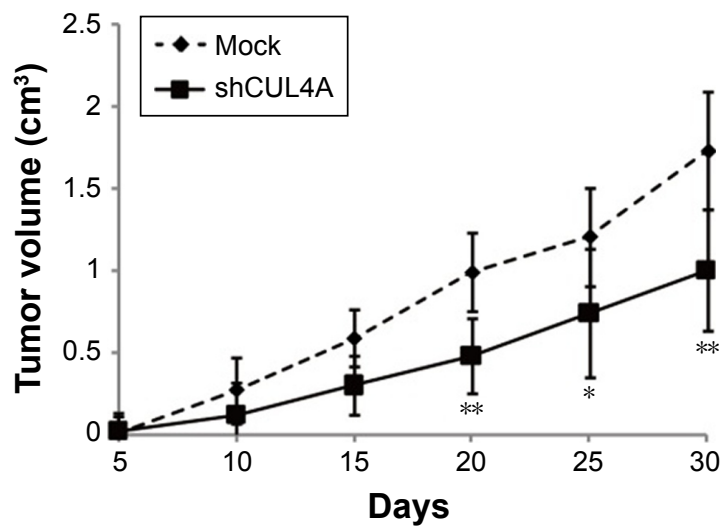

C

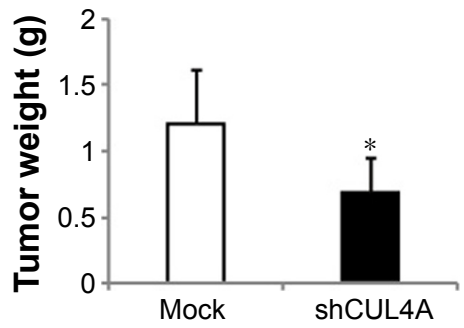

B

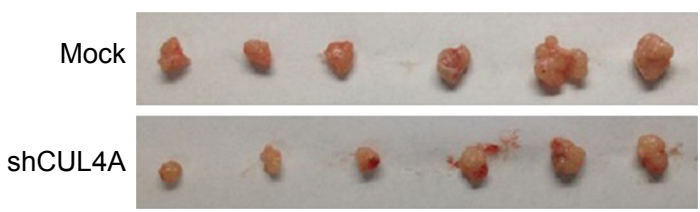

D

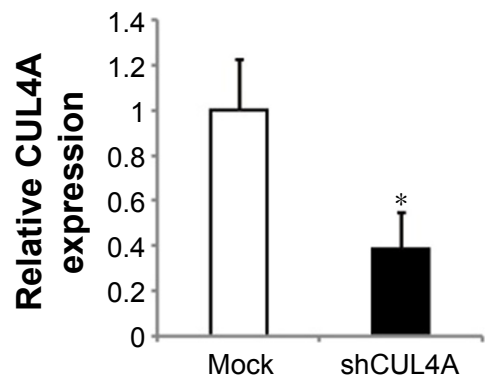

Figure 5 Knockdown of CUL4A suppresses tumor growth in vivo.

Notes: (A and B) Tumor volume was calculated every 5 days after the injection of HCT-II 6 cells with knockdown of CUL4A. Error bars indicate SD. (C) Tumor weight is represented as mean of tumor weights \pm SD. (D) qPCR analysis of CUL4A expression in tumor tissues formed from HCT-II6/mock and HCT-II6/shCUL4A. *P $\leq 0.05$, $* * P \leq 0.01$.

Abbreviations: qPCR, quantitative polymerase chain reaction; SD, standard deviation.

shRNA (Figure 5D). In conclusion, these data demonstrate that CUL4A promotes CRC tumorigenesis and knockdown of CUL4A has a negative effect on tumor growth in vivo.

\section{Discussion}

To the best of our knowledge, it is the first systematic study to evaluate the role CUL4A plays in CRC patients. Our study revealed that increased expression of CUL4A resulted in a more aggressive phenotype in CRC patients. Furthermore, CUL4A overexpression in CRC cell line HCT-116 induced proliferation and migration in vitro. Consistently, knockdown of CUL4A reversed these events. We also found the relation between CUL4A and EMT through CUL4A-mediated regulation of $\mathrm{H} 3 \mathrm{~K} 4 \mathrm{me} 3$, which results in transcriptional downregulation of epithelial marker E-cadherin and upregulation of mesenchymal markers $\mathrm{N}$-cadherin and vimentin expression. Generally, the present study revealed that CUL4A can be considered as a therapy target for CRC.

Abnormal gene expression changes are involved in tumorigenesis, which will induce a series of target gene alterations and subsequent biological changes and this cascade of events is crucial to tumorigenesis. ${ }^{26}$ Previous studies have provided evidence that CUL4A might serve as an oncogene in several kinds of cancers for the reason that CUL4A is highly expressed in malignant tumors, such as breast cancer, prostate cancer, NSCLC, and MPM relative to normal tissues. ${ }^{19-23}$ Deletion of CUL4A in mouse resulted in dramatically increased resistance to ultraviolet-induced skin carcinogenesis. ${ }^{27}$ Furthermore, as CUL4A ubiquitinates and degrades several well-known tumor suppressor genes p21, p27, p53, and DDB2, CUL4A can be considered an oncogene in certain contexts. ${ }^{15-18}$ Consistent with these previous studies, CUL4A overexpression promoted CRC cell line HCT-116 proliferation, migration, and invasion both in vitro and in vivo. Our study found a novel function of CUL4A in CRC metastasis through regulating EMT by H3K4me3 modulation.

\section{Conclusion}

In this study, we found that the levels of $\mathrm{H} 3 \mathrm{~K} 4 \mathrm{me} 3$ at the E-cadherin, N-cadherin, and vimentin gene promoters could be altered by CUL4A expression, which subsequently controlled their expression. However, we did not detect the influence of CUL4A expression on the methylation status of $\mathrm{H} 3 \mathrm{~K} 9, \mathrm{H} 3 \mathrm{~K} 27$, and other histone modifications. We conclude that CUL4A transcriptionally regulates 
E-cadherin, N-cadherin, and vimentin expression through regulation of $\mathrm{H} 3 \mathrm{~K} 4 \mathrm{me} 3$ and recruitment of $\mathrm{H} 3 \mathrm{~K} 4 \mathrm{me} 3$ to these gene promoters, and consequently promotes EMT. Although how CUL4A modulates H3K4me3 in CRC requires further clarification. In summary, our experiments provides evidence that CUL4A is both a potential therapeutic target and biomarker for CRC.

\section{Disclosure}

The authors report no conflicts of interest in this work.

\section{References}

1. Fitzmaurice C, Dicker D, Pain A, et al; Global Burden of Disease Cancer Collaboration. The global burden of cancer 2013. JAMA Oncol. 2015; 1(4):505-527.

2. Shen Z, Deng H, Fang Y, et al. Identification of the interplay between SOX9 and S100P in the metastasis and invasion of colon carcinoma Oncotarget. 2015;6(24):20672-20684.

3. Vincenzi B, Cremolini C, Sartore-Bianchi A, et al. Prognostic significance of K-Ras mutation rate in metastatic colorectal cancer patients. Oncotarget. 2015;6(31):31604-31612.

4. Yi C, Huang Y, Yu X, et al. Clinicopathologic distribution of KRAS and BRAF mutations in a Chinese population with colorectal cancer precursor lesions. Oncotarget. 2016;7(13):17265-17274.

5. Yang J, Zhang K, Song H, et al. Visfatin is involved in promotion of colorectal carcinoma malignancy through an inducing EMT mechanism. Oncotarget. 2016;7(22):32306-32317.

6. Wei Z, Ma W, Qi X, et al. Pinin facilitated proliferation and metastasis of colorectal cancer through activating EGFR/ERK signaling pathway. Oncotarget. 2016;7(20):29429-29439.

7. Brabletz T, Hlubek F, Spaderna S, et al. Invasion and metastasis in colorectal cancer: epithelial-mesenchymal transition, mesenchymalepithelial transition, stem cells and beta-catenin. Cells Tissues Organs. 2005;179(1-2):56-65.

8. Lamouille S, Xu J, Derynck R. Molecular mechanisms of epithelialmesenchymal transition. Nat Rev Mol Cell Biol. 2014;15(3):178-196.

9. Bernstein BE, Mikkelsen TS, Xie X, et al. A bivalent chromatin structure marks key developmental genes in embryonic stem cells. Cell. 2006; 125(2):315-326.

10. Zhong W, Feng H, Santiago FE, Kipreos ET. CUL-4 ubiquitin ligase maintains genome stability by restraining DNA-replication licensing. Nature. 2003;423(6942):885-889.

11. Lee J, Zhou P. Pathogenic role of the CRL4 ubiquitin ligase in human disease. Front Oncol. 2012;2:21.
12. Sugasawa K. The CUL4 enigma: culling DNA repair factors. Mol Cell. 2009;34(4):403-404.

13. Han J, Zhang H, Zhang H, Wang Z, Zhou H, Zhang Z. A Cul4 E3 ubiquitin ligase regulates histone hand-off during nucleosome assembly. Cell. 2013;155(4):817-829.

14. Hu J, Xiong Y. An evolutionarily conserved function of proliferating cell nuclear antigen for Cdt1 degradation by the Cul4-Ddb1 ubiquitin ligase in response to DNA damage. J Biol Chem. 2006;281(7):3753-3756.

15. Li B, Jia N, Kapur R, Chun KT. Cul4A targets p27 for degradation and regulates proliferation, cell cycle exit, and differentiation during erythropoiesis. Blood. 2006;107(11):4291-4299.

16. Nishitani H, Shiomi Y, Iida H, Michishita M, Takami T, Tsurimoto T. $\mathrm{CDK}$ inhibitor $\mathrm{p} 21$ is degraded by a proliferating cell nuclear antigencoupled Cul4-DDB1Cdt2 pathway during S phase and after UV irradiation. J Biol Chem. 2008;283(43):29045-29052.

17. Nag A, Bagchi S, Raychaudhuri P. Cul4A physically associates with MDM2 and participates in the proteolysis of p53. Cancer Res. 2004; 64(22):8152-8155.

18. Li J, Wang QE, Zhu Q. DNA damage binding protein component DDB1 participates in nucleotide excision repair through DDB2 DNAbinding and cullin 4A ubiquitin ligase activity. Cancer Res. 2006; 66(17):8590-8597.

19. Wang Y, Wen M, Kwon Y. CUL4A induces epithelial-mesenchymal transition and promotes cancer metastasis by regulating ZEB1 expression. Cancer Res. 2014;74(2):520-531.

20. Melchor L, Saucedo-Cuevas LP, Muñoz-Repeto I, et al. Comprehensive characterization of the DNA amplification at 13q34 in human breast cancer reveals TFDP1 and CUL4A as likely candidate target genes. Breast Cancer Res. 2009;11(6):R86.

21. Ren S, Xu C, Cui Z, et al. Oncogenic CUL4A determines the response to thalidomide treatment in prostate cancer. $J$ Mol Med (Berl). 2012; 90(10):1121-1132.

22. Wang Y, Zhang P, Liu Z, et al. CUL4A overexpression enhances lung tumor growth and sensitizes lung cancer cells to erlotinib via transcriptional regulation of EGFR. Mol Cancer. 2014;13:252.

23. Hung MS, Mao JH, Xu Z, et al. Cul4A is an oncogene in malignant pleural mesothelioma. J Cell Mol Med. 2011;15(2):350-358.

24. Jackson S, Xiong Y. CRL4s: the CUL4-RING E3 ubiquitin ligases. Trends Biochem Sci. 2009;34(11):562-570.

25. Higa LA, Wu M, Ye T, Kobayashi R, Sun H, Zhang H. CUL4-DDB1 ubiquitin ligase interacts with multiple WD40-repeat proteins and regulates histone methylation. Nat Cell Biol. 2006;8(11):1277-1283.

26. Oxnard GR, Binder A, Jänne PA. New targetable oncogenes in nonsmall-cell lung cancer. J Clin Oncol. 2013;31(8):1097-1104.

27. Liu L, Lee S, Zhang J, et al. CUL4A abrogation augments DNA damage response and protection against skin carcinogenesis. Mol Cell. 2009; 34(4):451-460.
OncoTargets and Therapy

\section{Publish your work in this journal}

OncoTargets and Therapy is an international, peer-reviewed, open access journal focusing on the pathological basis of all cancers, potential targets for therapy and treatment protocols employed to improve the management of cancer patients. The journal also focuses on the impact of management programs and new therapeutic agents and protocols on

\section{Dovepress}

patient perspectives such as quality of life, adherence and satisfaction. The manuscript management system is completely online and includes a very quick and fair peer-review system, which is all easy to use. Visit http://www.dovepress.com/testimonials.php to read real quotes from published authors. 